\title{
Subcellular protein overexpression to develop abiotic stress tolerant plants
}

\author{
Mohammad-Zaman Nouri ${ }^{1 *}$ and Setsuko Komatsu ${ }^{2 *}$ \\ 1 Plant Breeding Department, Rice Research Institute of Iran-Deputy of Mazandaran, Amol, Iran \\ ${ }^{2}$ National Institute of Crop Science, National Agriculture and Food Research Organization, Tsukuba, Japan
}

\author{
Edited by: \\ Pingfang Yang, Chinese Academy of \\ Sciences, China \\ Reviewed by: \\ Georgia Tanou, Aristotle University \\ of Thessaloniki, Greece \\ Hans-Peter Mock, Institute of Plant \\ Genetics and Crop Plant Reserach \\ (IPK), Germany \\ *Correspondence: \\ Mohammad-Zaman Nouri, Plant \\ Breeding Department, Rice \\ Research Institute of Iran-Deputy of \\ Mazandaran, Amol 46191-91951. \\ Iran. \\ e-mail:m.nouri@areo.ir \\ Setsuko Komatsu, National Institute \\ of Crop Science, National \\ Agriculture and Food Research \\ Organization, Kannondai 2-1-18, \\ Tsukuba 305-8518, Japan. \\ e-mail: skomatsu@affrc.go.jp
}

\begin{abstract}
Environmental stresses are major factors limiting growth and development of crops. Plants respond to the stresses through a wide range of reactions from morphological changes to alterations in the patterns of protein expression. Understanding the mechanisms involved in the stress response is the first step to develop abiotic stress tolerant crops. Proteomics is a powerful tool in evaluating regulated proteins in the cell under stress and it is an efficient technique in studying stress tolerant plants. Because of the nature of abiotic stress, intracellular compartments play a main role in the stress response. Subcellular proteins such as ion and water transporters, reactive oxygen species (ROS) scavengers, and the proteins related to signaling and transcriptional regulation are frequently reported as being involved in stress tolerance. Overexpression of stress-responsive protein through generation of transgenic plants is one the main practical approaches in production of tolerant plants. In this article, recent studies on transgenic plants overexpressing subcellular proteins are reviewed and the role of organelles and over-expressed proteins is classified.
\end{abstract}

Keywords: subcellular protein, transgenic, overexpression, abiotic stress

\section{INTRODUCTION}

Adverse environmental conditions threaten normal growth and development of plants. Abiotic stresses mainly including temperature extremes, drought, and salinity detrimentally affect plant growth and crop yield. It was reported that for most crops, abiotic stresses are leading to a reduction in the average yield by more than $50 \%$ (Bray et al., 2000). One-third of the world's population resides in water-stressed regions, and because of climate changes, water stress could become more frequent and severe in the future (Manavalan et al., 2009). Adverse abiotic stresses tend to occur together and they are almost never present individually in nature. On the other hand, plant cells likely follow similar mechanisms to cope with abiotic stresses. For instance, cold, drought, and salinity are well-known to the generation of reactive oxygen species (ROS) causing oxidative stress (Dat et al., 2000). Therefore, ROS scavenging genes are candidate in the generation of plants with tolerance to the stress.

At the cellular level, plants adopt a wide range of responses to cope with abiotic stress. The mechanism associated with sensing stress, transduction of stress signals into the cell is well-known, and it represents the initial reaction of plant cells to stress (Desikan et al., 2004). Stress signals are first encountered by the outer parts of the cell under a highly organized process for sensing environmental changes (Heino and Palva, 2003). ROS, which are formed by partial reduction of molecular oxygen during abiotic stress, are recognized as a signal to activate the defense response (Vranová et al., 2002). Transduction of the signal into the cell through cascades alters gene and protein expression, leading to physiological responses. Communication through intracellular compartments plays an important role in this process.

The major subcellular organelles and compartments in plant cells are nucleus, mitochondria, chloroplasts, endoplasmic reticulum (ER), Golgi apparatus, vacuoles, and plasma membrane. The intracellular organelles and their interactions during stressful conditions represent the primary defense response. In fact, communication between organelles and cytosolic and luminal proteins renders the protein composition of organelles dynamic (Agrawal et al., 2011). Most receptor proteins are located in the plasma membrane, and thus the plasma membrane is directly involved in stress sensing (Komatsu, 2008). Intracellular organelles with high capacity for production of ROS, such as mitochondria and chloroplasts are the primary sites for the production of this signaling molecule. ROS can also increase the effects of cellular damage (Dat et al., 2000; Van Breusegem et al., 2001). According to the role of cellular organelles and compartments, there are several key subcellular proteins involved in stress tolerance in plant cells.

Proteome analysis of cellular organelles under abiotic stress indicated that the accumulation of responsible proteins highly improves plant tolerance to the stress. Therefore, overexpression of related genes through the production of transgenic plants is a powerful technique to enhance stress tolerance in plants. Generation of tolerant transgenic plants against abiotic stresses has extensively been discussed in reviews (Wang et al., 2003; Bartels and Sunkar, 2005). However, classification of subcellularlocalized proteins and their related genes involved in abiotic stress 
response is scant. This review highlights the studies in recent decade on abiotic stress tolerant transgenic plants overexpressing subcellular proteins.

\section{ABIOTIC STRESS-RELATED PROTEINS IN PLANT CELL}

When the plant is exposed to abiotic stress, the proper function of the cell will be highly affected. Cell survival under stress condition directly depends on how the cell can adapt itself to the environment. Cellular defense mechanisms against abiotic stress are mainly controlled by the expression of responsible genes and proteins. Generally, many plants are susceptible to unfavorable environmental conditions. Therefore, overexpression of protective genes might help to reduce the deleterious effects of the stress. Generation of transgenic plants overexpressing the stressresponsive gene and protein will confer stress resistance leading to enhanced plant growth and productivity (Allen et al., 1997). Three major groups of genes are reported to be involved in the stress response. The first group are those that are involved in signaling cascades and in transcriptional regulation. The second group are those having a role in the protection of membranes and proteins and the third group are those involved in water and ion uptake and transport (Wang et al., 2003).

In order to know about major subcellular proteins conferring stress tolerance, it is important to understand the roles of organelles themselves in the cell. Organelles such as chloroplast and mitochondria are mainly responsible for metabolic processes including photosynthesis, photorespiration, oxidative phosphorylation, and the tricarboxylic acid cycle (Taylor et al., 2009). The plasma membrane is mainly involved in stress signal perception, transducing into the cell, and ion and water transport. The tonoplast is involved in ion balance and adjustment of water content. The nucleus has a variety of functions including transcriptional regulation, signaling, and gene regulation (Hossain et al., 2012). Despite the particular roles of organelles, their coordinated functions and interactions are important in the stress response. Hossain et al. (2012) classified the defense-related abiotic stress-responsive proteins into six major groups according to their functions. The protein groups are osmoprotectant regulators, ROS scavengers, ion transporters, water channels, molecular chaperones, and proteolysis-related proteins. Most of the protein groups are functionally attributed to organelles or compartments indicating the importance of subcellular proteins in response to stress conditions.

Apart from changes in the expression of organelle proteins, post-translational modifications (PTMs) of proteins are also known as a defense mechanism against abiotic stress. Redox proteomics is an increasingly emerging branch of proteomics aimed at identifying PTMs, particularly under stressful conditions. Recent proteome studies analyzed a number of redox-targets at organelles, which offered a view of the proteome modifications that are regulated by abiotic stress. For example, several proteins are identified as a target for S-nitrosylation in peroxisomes (Ortega-Galisteo et al., 2012) and mitochondria (Camejo et al., 2012) of pea plants under abiotic stress conditions. The modification of organelle proteins could regulate $\mathrm{H}_{2} \mathrm{O}_{2}$ level or modulate the respiratory and photorespiratory pathways in the plant.

\section{OVEREXPRESSING OF ORGANELLE PROTEINS IN TRANSGENIC PLANT IMPROVES TOLERANCE TO ABIOTIC STRESS}

Transgenic plants overexpressing the genes encoded subcellularlocalized proteins are classified (Table 1). Stress-responsive genes or proteins were arranged according to the localization of the overexpressed protein in the cell. Nucleus, chloroplast, plasma membrane, ER, mitochondria, and vacuole are the organelles and compartments in which the presence of overexpressed proteins was confirmed (Table 1). Among the organelles, near half $(42 \%)$ of the overexpressed proteins was localized in the nucleus (Figure 1A). This means that the genes encode nuclear proteins were highly considered in the generation of transgenic plants. According to the role of nucleus protein under abiotic stress, it can be postulated that the molecular mechanism of stress tolerance in most of the studied transgenic plants is based on transcriptional regulation, signaling, and gene regulation. Other overexpressed proteins were mainly localized in chloroplast (23\%), plasma membrane (13\%), and ER (10\%) (Figure 1A). This classification performs a preliminary ranking of the importance of organelles in response to abiotic stress, regardless of the function of overexpressed protein.

Classification of the transgenic plant species indicated that out of 30 overexpressed proteins, Arabidopsis, rice, and tobacco were applied in 16, 6, and 5 studies, respectively (Table 1). Interestingly, while overexpressed proteins of Arabidopsis and tobacco were localized in diverse organelles, the overexpressed proteins of rice were only localized in the nucleus and chloroplast. Bartels and Sunkar (2005) classified stress-responsive genes contributing to drought or salt tolerance in transgenic plants. They reported a similar order for a number of successful transgenic Arabidopsis, tobacco, and rice. This result indicates that despite great advances in the generation of transgenic plants, most of the transgenic plants are among the model plants.

Overexpression of a specific protein in transgenic plant confers a level of tolerance to the plant. A diverse range of stresses that are mainly related to unfavorable environmental conditions is classified as abiotic stress. Overexpressed gene or protein in most of the transgenic plants is contributing to salt $(36 \%)$ and drought $(29 \%)$ tolerance which means $65 \%$ of all studies were only focused on salt and drought tolerance. Tolerance to other abiotic stresses such as cold (13\%), oxidative (7\%), and heat (5\%) were relatively less reported (Figure 1B). Manavalan et al. (2009) described the importance of water stress at the present and in the future. Therefore, researchers are working more on the generation of salt and drought stress tolerant transgenic plants.

\section{EFFECTS OF PROTEIN OVEREXPRESSION ON CELLULAR MECHANISMS OF PLANT}

A wide range of responses has been reported in transgenic plants subjected to abiotic stress. Alterations in morphology, physiology, and cellular mechanisms of transgenic plants are summarized (Table 1). The articles listed in the table, mainly did not comprehensively characterize cellular responses to the stresses. Therefore, it is difficult to perform functional classification of the overexpressed genes or proteins. However, growth enhancement in transgenic plants compared to wild type can be 


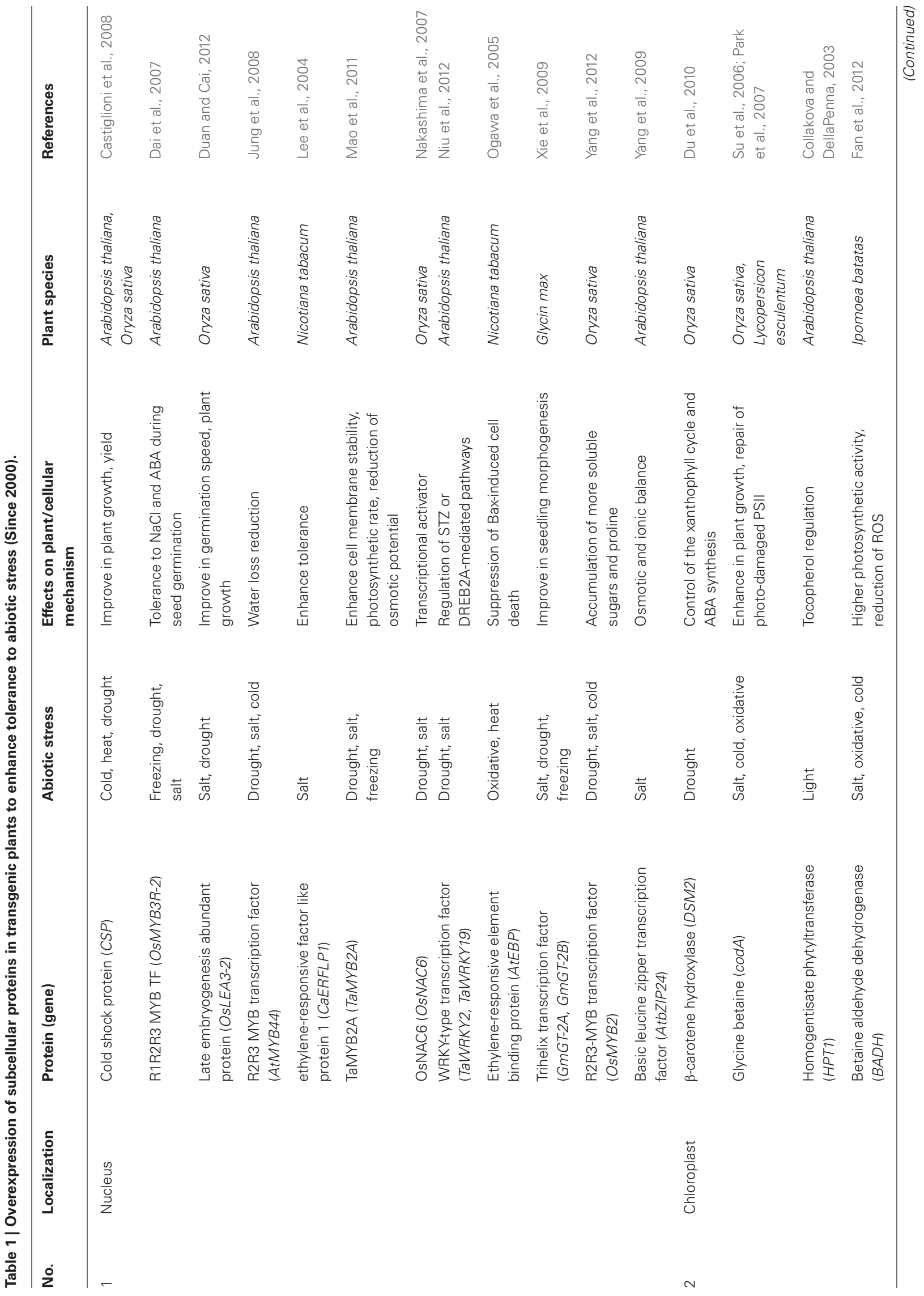




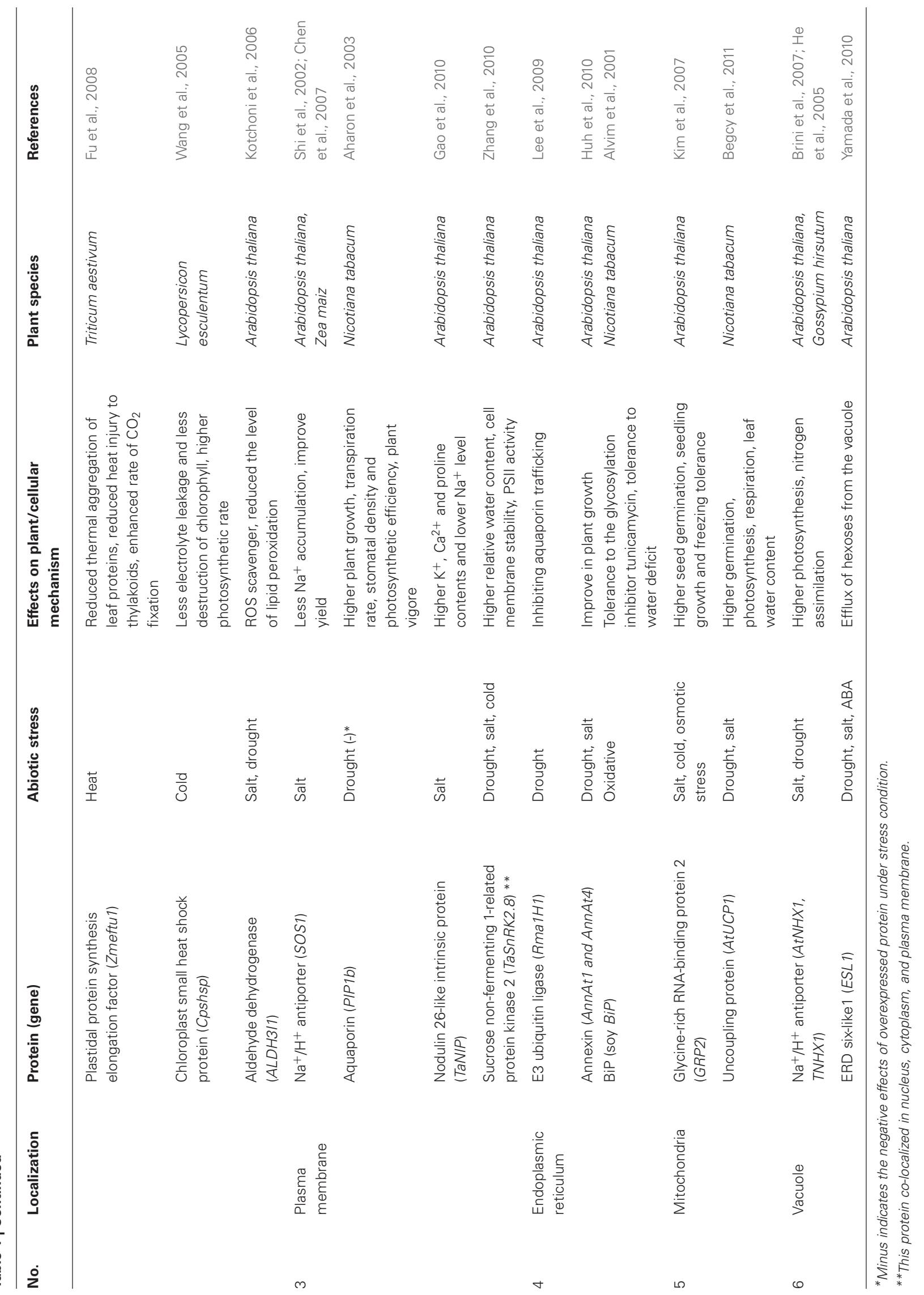




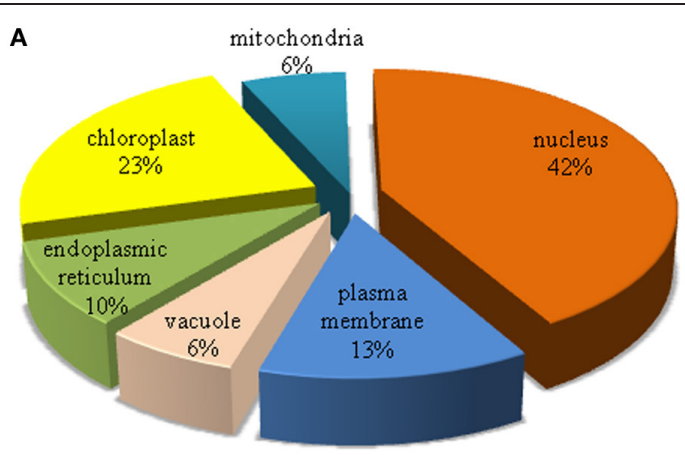

B

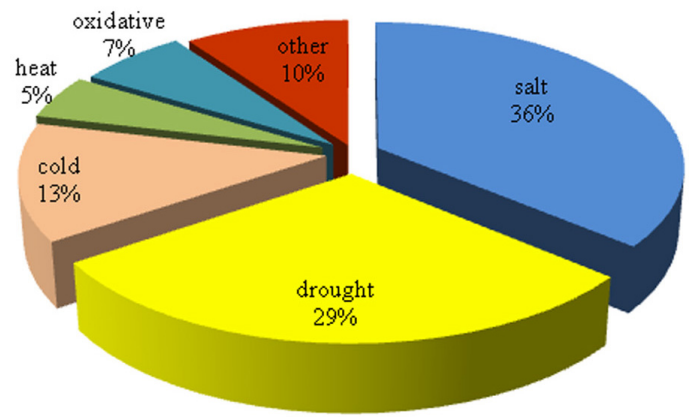

FIGURE 1 | Subcellular localization of overexpressed protein and type of abiotic stress is classified. (A) Percentage of organelles in which overexpressed proteins were localized. (B) Distribution of abiotic stresses in which transgenic plants were tolerant.

considered as a general morphological response. Improvement of the photosynthetic activity is another mechanism involved in stress tolerance. Proteins including MYB transcription factor (TaMYB2A) (Mao et al., 2011), glycine betaine (Park et al., 2007), betaine aldehyde dehydrogenase (Fan et al., 2012), chloroplast small heat shock protein (Wang et al., 2005), Sucrose nonfermenting 1-related protein kinase 2 (Zhang et al., 2010), and even $\mathrm{Na}^{+} / \mathrm{H}^{+}$antiporter (Brini et al., 2007) enhance photosynthetic activity. The proteins related to photosynthesis were not only localized in the chloroplast but also they were overexpressed in the other organelles.

Overexpression of some proteins in transgenic plant might have negative effects on plant tolerance to abiotic stress. Creissen et al. (1999) reported that in transgenic tobacco overexpressing chloroplast-targeted $\gamma$-glutamylcysteine synthetase $(\gamma$-ECS), foliar levels of glutathione were significantly raised. This protein as a major antioxidant in most aerobic organisms supposed

\section{REFERENCES}

Agrawal, G. K., Bourguignon, J., Rolland, N., Ephritikhine, G., Ferro, M., Jaquinod, M., et al. (2011). Plant organelle proteomics: collaborating for optimal cell function. Mass Spectrom. Rev. 30, 772-853.

Aharon, R., Shahak, Y., Wininger, S., Bendov, R., Kapulnik, Y., and Galili, G. (2003). Overexpression of a plasma membrane aquaporin in transgenic tobacco improves plant vigor under favorable growth conditions but not under drought or salt stress. Plant Cell 15, 439-447.

Allen, R. D., Webb, R. P., and Schake, S. A. (1997). Use of transgenic plants to study antioxidant defenses. Free Radic. Biol. Med. 23, 473-479.

to protect the photosynthetic apparatus from oxidative damage. Paradoxically, however, increased glutathione biosynthetic capacity in the chloroplast resulted in greatly enhanced oxidative stress possibly by failure of the chloroplast homeostatic mechanism (Creissen et al., 1999). Transgenic tobacco overexpressing aquaporin $(P I P 1 \quad b)$ is another example of the susceptibility of transgenic plant to abiotic stress. Aquaporin improves plant growth rate, transpiration rate, stomatal density, and photosynthetic efficiency under favorable growth conditions. However, overexpression of aquaporin reduced plant growth under drought stress because of rapid wilting (Aharon et al., 2003). Therefore, two main strategies in the generation of successful transgenic plants are; (1) consideration of the cellular and subcellular interactions of overexpressed protein; (2) selection of stress-specific target gene or protein according to the main role of the protein under stress condition.

\section{CONCLUSIONS AND FUTURE PERSECTIVE}

Abiotic stress is one of the main challenges in expansion of planting area worldwide. Application of omics technology such as proteomics, transcriptomics, and metabolomics together with bioinformatics are frequently reported in plant abiotic stress studies (Moumeni et al., 2011; Hakeem et al., 2012). Proteomics improved the efficiency of conventional breeding by identification of the stress-responsive proteins. Overexpression of specific stress-responsive protein through the generation of transgenic plant is an efficient technique which has been successfully applied in model plants. In this review, a survey of recent decade studies (from 2000) to generate transgenic plants overexpressing subcellular-localized proteins is presented. Classification of transgenic plants according to the organelle indicated that $42 \%$ of the overexpressed proteins were localized in the nucleus. Therefore, the nucleus functions such as transcriptional regulation, signaling, and gene regulation are more considered in the generation of the plants with tolerance to abiotic stress.

Furthermore, 23 out of the 30 stress-responsive proteins were overexpressed only in Arabidopsis, rice, or tobacco. This result suggests that despite great advances in the generation of transgenic plants, this technique is mainly applied in the model plants. Therefore, one of the challenges in the future would be adopting the technology of protein overexpression in economically relevant crops. However, our knowledge about cellular mechanisms against abiotic stress as a prerequisite needs to be improved. Rapidly developing omics technology has the potential to decipher the functions of cell and intracellular organelles against abiotic stress in future studies.

Alvim, F. C., Carolino, S. M., Cascardo, J. C., Nunes, C. C., Martinez, C. A., Otoni, W. C., et al. (2001). Enhanced accumulation of BiP in transgenic plants confers tolerance to water stress. Plant Physiol. 126, 1042-1054.

Bartels, D., and Sunkar, R. (2005). Drought and salt tolerance in plants. Crit. Rev. Plant Sci. 24, 23-58.
Begcy, K., Mariano, E. D., Mattiello, L., Nunes, A. V., Mazzafera, P., Maia, I. G., et al. (2011). An Arabidopsis mitochondrial uncoupling protein confers tolerance to drought and salt stress in transgenic tobacco plants. PLOS ONE 6:e23776. doi: 10.1371/journal.pone.0023776

Bray, E. A., Bailey Serres, J., and Weretilnyk, E. (2000). "Responses 
to abiotic stresses," in Biochemistry and Molecular Biology of Plant, eds W. Gruissem, B. Buchannan, and R. Jones (Rockville, MD: American Society of Plant Biologists), 158.

Brini, F., Hani, M., Mezghan, I., Berkowitz, G. A., and Masmoudi, K. (2007). Overexpression of wheat $\mathrm{Na}^{+} / \mathrm{H}^{+}$antiporter TNHX1 and $\mathrm{H}^{+}$-pyrophosphatase TVP1 improve salt- and drought-stress tolerance in Arabidopsis thaliana plants. J. Exp. Bot. 58, 301-308.

Camejo, D., Romero-Puertasm, M. D., Rodríguez-Serrano, M., Sandalio, L. M., Lázaro, J. J., Jiménez, A., et al. (2012). Salinity-induced changes in S-nitrosylation of pea mitochondrial proteins. J. Proteomics pii: S1874-391900782-391900788. doi: 10.1016/j.jprot.2012.12.003

Castiglioni, P., Warner, D., Bensen, R. J., Anstrom, D. C., Harrison, J., Stoecker, M., et al. (2008). Bacterial RNA chaperones confer abiotic stress tolerance in plants and improved grain yield in maize under water-limited conditions. Plant Physiol. 147, 446-455.

Chen, M., Chen, Q. J., Niu, X. J., Zhang, R., Li, H. Q., Xu, C. Y., et al. (2007). Expression of OsNHX1 gene in maize confers salt tolerance and promotes plant growth in the field. Plant Soil Environ. 53, 490-498.

Collakova, E., and DellaPenna, D. (2003). The role of homogentisate phytyltransferase and other tocopherol pathway enzymes in the regulation of tocopherol synthesis during abiotic stress. Plant Physiol. 133, 930-940.

Creissen, G., Firmin, J., Fryer, M., Kular, B., Leyland, N., Reynolds, H., et al. (1999). Elevated glutathione biosynthetic capacity in the chloroplasts of transgenic tobacco plants paradoxically causes increased oxidative stress. Plant Cell 11, 1277-1292.

Dai, X., Xu, Y., Ma, Q., Xu, W., Wang, T., Xue, Y., et al. (2007). Overexpression of an R1R2R3 MYB gene, OsMYB3R-2, increases tolerance to freezing, drought, and salt stress in transgenic Arabidopsis. Plant Physiol. 143, 1739-1751.

Dat, J., Vandenabeele, S., Vranová, E. Van Montagu, M., Inzé, D., and Van Breusegem, F. (2000). Dual action of the active oxygen species during plant stress responses. Cell. Mol. Life Sci. 57, 779-795.

Desikan, R., Hancock, J. T., and Neill, S. J. (2004). "Oxidative stress signalling," in Plant Responses to Abiotic Stress, eds H. Hirt and K. Shinozaki (Berlin: Springer-Verlag), 73-93.
Du, H., Wang, N., Cui, F., Li, X., Xiao, J., and Xiong, L. (2010). Characterization of the betacarotene hydroxylase gene DSM2 conferring drought and oxidative stress resistance by increasing xanthophylls and abscisic acid synthesis in rice. Plant Physiol. 154, 1304-1318.

Duan, J., and Cai, W. (2012). OsLEA3-2, an abiotic stress induced gene of rice plays a key role in salt and drought tolerance. PLOS ONE 7:e45117. doi: 10.1371/journal.pone.0045117

Fan, W., Zhang, M., Zhang, H., and Zhang, P. (2012). Improved tolerance to various abiotic stresses in transgenic sweet potato (Ipomoea batatas) expressing spinach betaine aldehyde dehydrogenase. PLoS ONE 7:e37344. doi: 10.1371/journal.pone.0037344

$\mathrm{Fu}$, J., Momcilović, I., Clemente, T. E., Nersesian, N., Trick, H. N., and Ristic, Z. (2008). Heterologous expression of a plastid EF-Tu reduces protein thermal aggregation and enhances $\mathrm{CO} 2$ fixation in wheat (Triticum aestivum) following heat stress. Plant Mol. Biol. 68, 277-288.

Gao, Z., He, X., Zhao, B., Zhou, C., Liang, Y., Ge, R., et al. (2010). Overexpressing a putative aquaporin gene from wheat, TaNIP, enhances salt tolerance in transgenic Arabidopsis. Plant Cell Physiol. 51, 767-775.

Hakeem, K. R., Chandna, R., Ahmad, P., Iqbal, M., and Ozturk, M. (2012). Relevance of proteomic investigations in plant abiotic stress physiology. OMICS 16, 621-635.

He, C., Yan, J., Shen, G., Fu, L., Holaday, A. S., Auld, D., et al. (2005). Expression of an Arabidopsis vacuolar sodium/proton antiporter gene in cotton improves photosynthetic performance under salt conditions and increases fiber yield in the field. Plant Cell Physiol. 46, 1848-1854.

Heino, P., and Palva, E. T. (2003) "Signal transduction in plant cold acclimation," in Plant Responses to Abiotic Stress, eds H. Hirt and K. Shinozaki (Berlin: Springer-Verlag), 151-186.

Hossain, Z., Nouri, M. Z., and Komatsu, S. (2012). Plant cell organelle proteomics in response to abiotic stress. J. Proteome Res. 11, 37-48.

Huh, S. M., Noh, E. K., Kim, H. G., Jeon, B. W., Bae, K., Hu, H. C., et al. (2010). Arabidopsis annexins AnnAt1 and AnnAt4 interact with each other and regulate drought and salt stress responses. Plant Cell Physiol. 51, 1499-1514.

Jung, C., Seo, J. S., Han, S. W. Koo, Y. J., Kim, C. H., Song, S. I., et al. (2008). Overexpression of AtMYB44 enhances stomatal closure to confer abiotic stress tolerance in transgenic Arabidopsis. Plant Physiol. 146, 623-635.

Kim, J. Y., Park, S. J., Jang, B., Jung, C. H., Ahn, S. J., Goh, C. H., et al. (2007). Functional characterization of a glycine-rich RNA-binding protein 2 in Arabidopsis thaliana under abiotic stress conditions. Plant J. 50, 439-451.

Komatsu, S. (2008). Plasma membrane proteome in Arabidopsis and rice. Proteomics 8, 4137-4145.

Kotchoni, S. O., Kuhns, C., Ditzer, A., Kirch, H. H., and Bartels, D. (2006). Over-expression of different aldehyde dehydrogenase genes in Arabidopsis thaliana confers tolerance to abiotic stress and protects plants against lipid peroxidation and oxidative stress. Plant Cell Environ. 29, 1033-1048.

Lee, H. K., Cho, S. K., Son, O., Xu, Z. Hwang, I., and Kim, W. T. (2009). Drought stress-induced RmalH1, a RING membrane-anchor E3 ubiquitin ligase homolog, regulates aquaporin levels via ubiquitination in transgenic Arabidopsis plants. Plant Cell 21, 622-641.

Lee, J. H., Hong, J. P., Oh, S. K., Lee, S., Choi, D., and Kim, W. T. (2004). The ethylene-responsive factor like protein 1 (CaERFLP1) of hot pepper (Capsicum annuum L.) interacts in vitro with both GCC and DRE/CRT sequences with different binding affinities: possible biological roles of CaERFLP1 in response to pathogen infection and high salinity conditions in transgenic tobacco plants. Plant Mol. Biol. 55, 61-81.

Manavalan, L. P., Guttikonda, S. K. Tran, L. S., and Nguyen, H. T. (2009). Physiological and molecular approaches to improve drought resistance in soybean. Plant Cell Physiol. 50, 1260-1276.

Mao, X., Jia, D., Li, A., Zhang, H. Tian, S., Zhang, X., et al. (2011). Transgenic expression of TaMYB2A confers enhanced tolerance to multiple abiotic stresses in Arabidopsis. Funct. Integr. Genomics 11, 445-465. Moumeni, A., Satoh, K., Kondoh, H. Asano, T., Hosaka, A., Venuprasad, R., et al. (2011). Comparative analysis of root transcriptome profiles of two pairs of drought-tolerant and susceptible rice near-isogenic lines under different drought stress. BMC Plant Biol. 11:174. doi: 10.1186/1471-2229-11-174
Nakashima, K., Tran, L. S., Van Nguyen, D., Fujita, M., Maruyama, K., Todaka, D., et al. (2007). Functional analysis of a NACtype transcription factor OsNAC6 involved in abiotic and biotic stressresponsive gene expression in rice. Plant J. 51, 617-630.

Niu, C. F., Wei, W., Zhou, Q. Y., Tian, A. G., Hao, Y. J., Zhang, W. K., et al. (2012). Wheat WRKY genes TaWRKY2 and TaWRKY19 regulate abiotic stress tolerance in transgenic Arabidopsis plants. Plant Cell Environ. 35, 1156-1170.

Ogawa, T., Pan, L., Kawai-Yamada, M., Yu, L. H., Yamamura, S., Koyama, T., et al. (2005). Functional analysis of Arabidopsis ethylene-responsive element binding protein conferring resistance to $\mathrm{Bax}$ and abiotic stress-induced plant cell death. Plant Physiol. 138, 1436-1445.

Ortega-Galisteo, A. P., RodríguezSerrano, M., Pazmiño, D. M., Gupta, D. K., Sandalio, L. M., and Romero-Puertas, M. C. (2012). S-Nitrosylated proteins in pea (Pisum sativum L.) leaf peroxisomes: changes under abiotic stress. J. Exp. Bot. 63, 2089-2103.

Park, E. J., Jeknić, Z., Pino, M. T., Murata, N., and Chen, T. H. (2007). Glycinebetaine accumulation is more effective in chloroplasts than in the cytosol for protecting transgenic tomato plants against abiotic stress. Plant Cell Environ. 30, 994-1005.

Shi, H., Lee, B. H., Wu, S. J., and Zhu, J. K. (2002). Overexpression of a plasma membrane $\mathrm{Na}^{+} / \mathrm{H}^{+}$ antiporter gene improves salt tolerance in Arabidopsis thaliana. Nat. Biotechnol. 21, 81-85.

Su, J., Hirji, R., Zhang, L., He, C., Selvaraj, G., and Wu, R. (2006). Evaluation of the stress-inducible production of choline oxidase in transgenic rice as a strategy for producing the stress-protectant glycine betaine. J. Exp. Bot. 57, 1129-1135.

Taylor, N. L., Tan, Y. F., Jacoby, R. P., and Millar, A. H. (2009). Abiotic environmental stress induced changes in the Arabidopsis thaliana chloroplast, mitochondria and peroxisome proteomes. J. Proteomics 72, 367-378.

Van Breusegem, F., Vranová, E., Dat, J. F., and Inze, D. (2001). The role of active oxygen species in plant signal transduction. Plant Sci. 161, 405-414.

Vranová, E., Inzé, D., and Van Breusegem, F. (2002). Signal transduction during oxidative stress. J. Exp. Bot. 53, 1227-1236.

Wang, L., Zhao, C. M., Wang, Y. J., and Liu, J. (2005). Overexpression of 
chloroplast-localized small molecular heat-shock protein enhances chilling tolerance in tomato plant. J. Plant Physiol. Mol. Biol. 31, 167-174.

Wang, W., Vinocur, B., and Altman, A. (2003). Plant responses to drought, salinity and extreme temperatures: towards genetic engineering for stress tolerance. Planta 218, $1-14$.

Xie, Z. M., Zou, H. F., Lei, G., Wei, W., Zhou, Q. Y., Niu, C. F., et al. (2009). Soybean trihelix transcription factors GmGT-2A and GmGT-2B improve plant tolerance to abiotic stresses in transgenic Arabidopsis. PLoS ONE 4:e6898. doi: 10.1371/journal.pone.0006898
Yamada, K., Osakabe, Y., Mizoi, J., Nakashima, K., Fujita, Y., Shinozaki, K., et al. (2010). Functional analysis of an Arabidopsis thaliana abiotic stress-inducible facilitated diffusion transporter for monosaccharides. J. Biol. Chem. 285, 1138-1146.

Yang, A., Dai, X., and Zhang, W. H. (2012). A R2R3-type MYB gene, OsMYB2, is involved in salt, cold, and dehydration tolerance in rice. J. Exp. Bot. 63, 2541-2556.

Yang, O., Popova, O. V., Süthoff, U., Lüking, I., Dietz, K. J., and Golldack, D. (2009). The Arabidopsis basic leucine zipper transcription factor AtbZIP24 regulates complex transcriptional networks involved in abiotic stress resistance. Gene 436, 45-55.

Zhang, H., Mao, X., Wang, C., and Jing, R. (2010). Overexpression of a common wheat gene TaSnRK2.8 enhances tolerance to drought, salt and low temperature in Arabidopsis. PLoS ONE 5:e16041. doi: 10.1371/journal.pone.0016041

Conflict of Interest Statement: The authors declare that the research was conducted in the absence of any commercial or financial relationships that could be construed as a potential conflict of interest.

Received: 23 November 2012; paper pending published: 13 December 2012; accepted: 02 January 2013; published online: 21 January 2013.

Citation: Nouri $M-Z$ and Komatsu $S$ (2013) Subcellular protein overexpression to develop abiotic stress tolerant plants. Front. Plant Sci. 4:2. doi 10.3389/fpls.2013.00002

This article was submitted to Frontiers in Plant Proteomics, a specialty of Frontiers in Plant Science.

Copyright (c) 2013 Nouri and Komatsu. This is an open-access article distributed under the terms of the Creative Commons Attribution License, which permits use, distribution and reproduction in other forums, provided the original authors and source are credited and subject to any copyright notices concerning any third-party graphics etc. 